\title{
DETECÇÃO DE IMUNOGLOBULINAS IgG, IgM E IgA ANTT-TOXOPLASMA GONDII NO SORO, LÍQUOR E SALIVA DE PACIENTES COM SÍNDROME DA IMUNODEFICIÊNCIA ADQUIRIDA E NEUROTOXOPLASMOSE
}

\author{
Aercio Sebastião Borges¹, José Fernando de Castro Figueiredo²
}

\begin{abstract}
RESUMO - Estudamos 55 pacientes com sindrome da imunodeficiência adquirida (SIDA) e neurotoxoplasmose (grupo 1); 37 pacientes com SIDA e comprometimento neurológico por outra etiologia (grupo 2) e 18 indivíduos anti-HIV negativoscom manifestações neurológicas (grupo 3), pesquisando lgG, IgA e lgM anti-Toxoplasma gondii, no soro, líquor e saliva, utilizando teste ELISA, para fins diagnósticos. O valor preditivo negativo do teste para o encontro de lgG no soro foi $100 \%$ e no líquor, $92,4 \%$. Não houve diferença entre os trêsgruposquanto aos anticorpos IgA neste material. Para IgA, no líquor, o teste alcançou $72,7 \%$ de especificidade $(p \varangle 0,05)$. Na saliva, apenaso encontro de lgG mostrou correlação como diagnóstico de neurotoxoplasmose. Enfatizamos que a ausência de anticorpos lgG anti-T. gondii no soro e líquor depõe fortemente contra o diagnóstico de neurotoxoplasmose e que imunoglobulinas IgA específicas no líquor e lgG na saliva podem representar dois marcadores auxiliares para o diagnóstico diferencial da encefalite toxoplásmica na SIDA.
\end{abstract}

PALAVRAS-CHAVE: SIDA, neurotoxoplasmose, anticorpos, soro, líquor, saliva.

\begin{abstract}
Detection of anti-Toxoplasma gondii IgG, IgM and IgA immunoglobulins in the serum, cerebrospinal fluid and saliva of patients with acquired immunodeficiency syndrome and neurotoxoplasmosis

ABSTRACT - We studied 55 patientswith acquired immunodeficiency syndrome (AIDS) and neurotoxoplasmosis (group 1), 37 patients with AIDS and neurological involvement due to another etiology (group 2) and 18 anti-HIV-negative individuals with neurological manifestations, by searching for anti-T. gondii IgG, IgA and IgM immunoglobulins in serum, cerebrospinal fluid (CSF)and saliva, using ELISA. The negative predictive value of the test for IgG in serum was $100 \%$ and in CSF, $92.4 \%$. There was no difference among the three groupsstudied regarding IgA in serum. For IgA, in CSF the test reached $72.7 \%$ specificity $(p<0.05)$. In saliva, only the detection of IgG wasfound to be correlated with a diagnosis of neurotoxoplasmosis. We emphasize that the absence of anti-T. gondii IgG antibodies in serum and CSF strongly indicates the absence of a diagnosis of neurotoxoplasmosis and that specific IgA immunoglobulins in CSF and IgG in saliva may represent two auxiliary markers for the differential diagnosis of toxoplasmic encephalitis in AIDS.
\end{abstract}

KEY WORDS: AIDS, neurotoxoplasmosis, antibodies, serum, cerebrospinal fluid, saliva.

A neurotoxoplasmose permanece como uma das principais causas de encefalite focal em pacientes com síndrome da imunodeficiência adquirida (SIDA). A doença resulta, na maioria dos casos, de reativação de infecção latente, com prevalência de 3\% a $50 \%$, de acordo com a área geográfica, e apresenta elevada morbi-mortalidade, se não reconhecida e tratada precocemente $\mathrm{e}^{1-3}$. O risco estimado de reativação a nível de sistema nervoso central (SNC) de pacientes com SIDA e infecção crônica varia de $12 \%$ a $47 \% \%^{4,5}$. O diagnóstico precoce da neurotoxoplasmose, nestes casos, tem sido limitado por falta de métodos propedêuticos não invasivos, de maior sensibilidade e especificidade. Manifesta-se clinicamente de forma semelhante às outras complicações oportunistas que acometem o SNC destes pacientes, taiscomo linfoma primário do SNC, encefalites virais ou fúngicas, reativação da doença de Chagas e neurotuberculose e, não raro, ocorrem concomitantemente duas ou mais infecções oportunistas no

\footnotetext{
${ }^{1}$ Centro de Ciências Biomédicas da Faculdade de Medicina da Universidade Federal de Uberlândia-UFU, Uberlândia MG, Brasil; ' 2 Divisão de Moléstias Infecciosase Tropicais do Departamento de Clínica Médica da Faculdade de Medicina de Ribeirão Preto da Universidade de São Paulo (USP), Ribeirão Preto SP, Brasil.
}

Recebido 7 Abril 2004, recebido na forma final 25 Junho 200. Aceito 5 Agosto 2004.

Dr. Aércio S Borges - Rua Niterói 1465 - 38406-017 Uberlândia MG - Brasil. E-mail: aerciosb@uol.com.br 
mesmo sítio, dificultando a confirmação diagnósti$\mathrm{ca}^{6-9}$. Os estudos de imagens, como a tomografia computadorizada (TC) de crânio e a ressonância magnética (RM), são de grande valor diagnóstico ao mostrarem lesõesisodensas ou hipodensas, únicas ou múltiplas, com efeito de massa e que captam o contraste de forma anelar ou nodular. Tais achados são encontrados em cerca de $90 \%$ dos casos, sendo bastante sugestivos de neurotoxoplasmose, porém não são patognomônicos ${ }^{10}$.

O perfil sorológico dos pacientes com SIDA e neurotoxoplasmose é semelhante ao da população geral, com infecção inativa. Anticorpos da classe IgM anti-Toxoplasma gondii não são habitualmente detectadose os da classe lgG não discriminam infecção latente de infecção ativa ${ }^{11,12}$. Taisanticorpos têm sido demonstrados, também, na saliva de indivíduos com toxoplasmose aguda e não em pacientes com infecção latente. A pesquisa de imunoglobulinas IgA no soro, um marcador de infecção aguda, apresenta resultados conflitantes em pacientescom neurotoxoplasmose e sua demonstração na saliva não se mostrou útil para diferenciação entre infecção recente e crônica ${ }^{13-15}$. Os achadosliquóricossão inespecíficos e e encontro de anticorpos no líquor deve ser interpretado com cautela, poisa sua presença pode significar apenastranferência passiva do soro para o SNC ${ }^{11,16}$.

Na maioria dos casos, portanto, o diagnóstico da neurotoxoplasmose é presuntivo, baseado nos achados de TC ou de RM e na presença de anticorpos IgG espećficosno soro, uma vez que em apenas $3 \%$ - $6 \%$ dos casos de reativação não se detecta tais anticorpos ${ }^{12,17,18}$. A confirmação se faz diante da resposta à terapêutica empiricamente instituída, que ocorre, em geral, entre 7 e 14 dias $^{10,19,20}$. Desta forma, em áreascomalta prevalência da toxoplas mose, tais pacientes podem ser expostos desnecessariamente aos ef eitosadversos do tratamento específico, em até $40 \%$ dos $\operatorname{casos}^{12,21,22}$.

O objetivo deste estudo foi avaliar a resposta imune humoral anti-T. gondii, através da pesquisa de anticorpos das classes IgM, IgA e IgG no soro, líquor e saliva, utilizando o ensaio imunoenzimático (EUSA), para fins diagnósticos da neurotoxoplasmose, em pacientes com SIDA.

\section{MÉTODO}

Foram estudados 110 pacientes, sendo 92 infectados pelo vírus da imunodeficiência humana (HIV-1) (teste ELISA - Abbot- $2^{\mathrm{a}}$. Geração e teste de aglutinação de partículas de gelatina - SerodiaR ${ }^{R}$ Fujirebio INC., Tokyo, Japan, para confirmação), com diagnóstico de SIDA, segundo critérios estabelecidos pelos CDC (Centers for Disease Control) ${ }^{23}$ e 18 não infectados. Foram considerados para o estudo pacientesadultos, com idade superior a 12 anos, de ambosossexos, que apresentavam manifestações clínicas de doença neurológica.

Os pacientes foram divididos em 3 grupos. $O$ grupo 1 compreendia 55 pacientes com SIDA e diagnóstico presuntivo de neurotoxoplasmose; o grupo 2, 37 pacientes com SIDA, sendo 19 com neurocriptococose, 3 com neurotuberculose, 2 com meningite bacteriana, 1 com complexo demencial relacionado à SIDA, diagnóstico estabelecido após exclusão de outras patologias, 2 com alterações hidroeletrolícase ácido-básicas com repercussão no SNC e, para os 10 restantes, a etiologia do quadro neurológico não ficou estabelecida. No grupo 3, 18 pacientesanti-HIV negativos: 8 com meningite bacteriana, 4 com diagnóstico de meningite linfomonocitária, 2 com neurocriptococose, 1 com paracoccidioidomicose no SNC, 1 com síndrome de Guillain-Barré, 1 com hemorragia sub-aracnóidea e o último paciente foi avaliado para investigação de cefaléia.

O diagnóstico presuntivo de neurotoxoplasmose foi estabelecido baseando-se no quadro dínico, nosachados tomográficos de lesões ocupando espaço, únicasou múltiplas, com efeito de massa e reforço anelar ou nodular após injeção do contraste e que apresentaram melhora, dínica e/ou radiológica, com a terapêutica específica com sulfadiazina ( $4 \mathrm{~g} / \mathrm{dia}$ ) ou clindamicina $(2,7 \mathrm{~g} / \mathrm{dia}$ ) associadas à pirimetamina $(50 \mathrm{mg} / \mathrm{dia})$.

A pesquisa de anticorpos anti-T. gondii, no soro, líquor e saliva, foi realizada através do ensaio imunoenzimático (ELISA), não competitivo, segundo Voller e cols. ${ }^{24}$ empregando placas de microtitulação de poliestireno (Dynatech) e sorosanti-imunoglobulina humana conjugados com peroxidase (Sigma Chemical Co., St. Louis, MO., USA). Os resultados foram analisados por comparação com osobtidosem amostras de pacientes infectados (controle positivo) e não infectados (controle negativo) pelo T. gondii. Foram consideradas reagentes as amostrasque apresentaram densidade óptica (D.O.) pelo menos 2,1 vezes maior do que a média aritmética das D.O. de duas amostras controles positivas (cut off).

Osdados foram processados através do programa Epi Info versão 6,02, 10/94. Para a análise de associações entre as variáveisfoi utilizado o teste do Qui-quadrado, sendo considerados significativos os valores de $\mathrm{p}$ menoresque 0,05 .

O estudo foi aprovado pela Comissão de Ética Médica em Pesquisa da Faculdade de Medicina de Ribeirão Preto-USP, com consentimento obtido dos pacientes ou de um familiar.

\section{RESULTADOS}

Todos os pacientes do grupo $1(100 \%), 90 \%$ do grupo 2 e $66,7 \%$ do grupo 3 tinham evidências de infecção crônica pelo T. gondii, pela presença de anticorpos IgG, específicos, no soro. O valor pre- 
ditivo negativo (VPN) do teste foi de $100 \%$ para o diagnóstico da neurotoxoplasmose na SIDA. Não houve diferença entre os 3 gruposestudados, quanto à pesquisa de anticorpos IgM e lgA, anti-T.gondii, no soro (Tabela 1).

Anticorpos da classe lgG foram encontrados no líquor de $98 \%$ dos pacientes do grupo $1,65,7 \%$ do grupo 2 e de $62,5 \%$ dospacientes do grupo 3 , alcançando $98 \%$ de sensibilidade e VPN de $92,4 \%$ para o diagnóstico diferencial da neurotoxoplasmose em pacientes com SIDA. Quanto à presença de IgM, neste fluido, não houve diferença entre os 3 grupos avaliados. Imunoglobulinas IgA foram encontradas em $50 \%$ dos pacientes do grupo 1 , em $27,3 \%$, do grupo 2 e em $6,3 \%$ dos pacientes do grupo 3, alcançando $72 \%$ de especificidade e valor preditivo positivo (VPP) de 71,8 ( $p=0,04)$ (Tabela 2).

Na saliva, apenaso encontro de lgG anti-T.gondii mostrou correlação com o diagnóstico de neurotoxoplasmose (Tabela 3).

\section{DISCUSSÃO}

A neurotoxoplasmose ainda é a principal causa de lesão expansiva no SNC de pacientes com SIDA, embora sua incidência venha decrescendo nosúltimos anos com o advento da terapia antiretroviral potente e o uso rotineiro da profilaxia com sulfametoxazol-trimetroprima, quando a contagem de células CD4+cai abaixo de 200/mm $3,12,25$, aumentando a necessidade de se estabelecer com maisacurácia tal diagnóstico. $O$ diagnóstico precoce da encefalite pelo T. gondii é presuntivo. A apresentação clínica, a análise do líquor e os resultados dos estudosradiológicos, podem se confundir com os de outras patologias que acometem o SNC destes pacientes. O diagnóstico etiológico definitivo de tais lesões pode requerer técnicas sofisticadas ou procedimentos invasivos não isentos de risco, sendo, então, estabelecido com base na resposta terapêutica empiricamente instituída, que ocorre, em geral, dentro dos primeiros 10 dias $^{10,20}$.

O valor dostestes sorológicos para deteç̧ão de anticorpos específicos circulantes, nestes casos, é discutível, uma vez que a doença resulta, comumente, de reativação da infecção latente, durante fase avançada de imunossupressão, sendo o perfil sorológico destes pacientes semel hante ao da população geral com infecção crônica, ou seja, habitualmente não se detectam anticorpos $\lg \mathrm{M}^{12,26}$. Alguns estudos, entretanto, detectaram tais anticorpos, através de diferentes técnicas, sobretudo em áreas de elevada prevalência da infecção pelo T. gondii, sem, contudo, definir seu valor diagnóstico ${ }^{17,27}$. No presente estudo, não encontramos diferença entre os 3 gruposavaliadosquanto à detecção de anticorpos da classe lgM no soro, líquor

Tabela 1. Resultados do teste ELISA no soro, segundo os diferentes grupos de pacientes estudados.

\begin{tabular}{|c|c|c|c|c|c|c|c|c|c|}
\hline \multirow[t]{2}{*}{ Elisa } & \multicolumn{3}{|c|}{ Grupo $1(n=54)^{*}$} & \multicolumn{3}{|c|}{ Grupo 2(n=30)* } & \multicolumn{3}{|c|}{ Grupo 3(n=15)* } \\
\hline & $\lg G$ & $\lg M$ & $\operatorname{Ig} A$ & $\lg G$ & $\lg M$ & $\lg A$ & $\lg G$ & $\lg M$ & $\lg A$ \\
\hline Positivo & 54 & 36 & 45 & 27 & 22 & 21 & 10 & 13 & 5 \\
\hline$\%$ & $100 \%$ & $66,7 \%$ & $83,3 \%$ & $90 \%$ & $73,3 \%$ & $70 \%$ & $66,7 \%$ & $86,7 \%$ & $33,3 \%$ \\
\hline
\end{tabular}

Tabela 2. Resultados do teste ELISA no líquor, segundo os diferentes grupos de pacientes estudados.

\begin{tabular}{|c|c|c|c|c|c|c|c|c|c|}
\hline \multirow[t]{2}{*}{ Elisa } & \multicolumn{3}{|c|}{ Grupo 1} & \multicolumn{3}{|c|}{ Grupo 2} & \multicolumn{3}{|c|}{ Grupo 3} \\
\hline & $\lg G$ & $\lg M$ & $\lg A$ & $\lg G$ & $\lg M$ & $\lg A$ & $\lg G$ & $\lg M$ & $\lg A$ \\
\hline Positivo/n* & $50 / 51$ & $13 / 51$ & $23 / 46$ & 23/35 & $5 / 35$ & $9 / 33$ & $10 / 16$ & $6 / 16$ & $1 / 16$ \\
\hline$\%$ & $98 \%$ & $25,5 \%$ & $50 \%$ & $65,7 \%$ & $14,3 \%$ & $27,3 \%$ & $62,5 \%$ & $37,5 \%$ & $6,3 \%$ \\
\hline
\end{tabular}

*Número de amostras em que o teste foi realizado.

Tabela 3. Resultados do teste ELISA na saliva, segundo os diferentes grupos de pacientes estudados.

\begin{tabular}{|c|c|c|c|c|c|c|c|c|c|}
\hline \multirow[t]{2}{*}{ Elisa } & \multicolumn{3}{|c|}{ Grupo 1} & \multicolumn{3}{|c|}{ Grupo 2} & \multicolumn{2}{|c|}{ Grupo 3} & \multirow[b]{2}{*}{$\lg A$} \\
\hline & $\lg G$ & $\lg M$ & $\lg A$ & $\lg G$ & $\lg M$ & $\lg A$ & $\lg G$ & $\lg M$ & \\
\hline Positivo/n* & $46 / 48$ & $32 / 48$ & $27 / 46$ & $17 / 27$ & $14 / 27$ & $10 / 27$ & $6 / 13$ & $10 / 13$ & $7 / 13$ \\
\hline$\%$ & $95,8 \%$ & $66,7 \%$ & $58,7 \%$ & $63 \%$ & $51,9 \%$ & $37 \%$ & $46,2 \%$ & $76,9 \%$ & $53,8 \%$ \\
\hline
\end{tabular}

* Número de amostras em que o teste foi realizado. 
ou na saliva, não sendo útil, portanto, para o diagnóstico diferencial da encefalite toxoplásmica na SIDA.

A presença de IgG anti- T.gondii no soro não é um marcador diagnóstico de neurotoxoplasmose, porém sua ausência afastou o diagnóstico em 100\% dosnossoscasos. Outrosautores referem, também, que em menos de $3 \%$ a $6 \%$ doscasos de encefalite toxoplásmica não se detectam evidências de infecção pregressa, ${ }^{8,12,17}$. Em contraste, o valor diagnóstico das imunoglobulinas lgA, importantes marcadores de toxoplasmose aguda e congênita, na reativação da toxoplasmose cerebral não é bem estabelecido, com algunsestudosmostrando resultados conflitantes $^{13,14,16}$. Não encontramos correlação entre sua positividade no soro e o diagnóstico de neurotoxoplasmose, comportando-se como osanticorpos IgM na reativação da toxoplasmose cerebral.

Tem-se demonstrado que aproximadamente $50 \%$ dos pacientes com encefalite pelo T. gondii irão apresentar anticorpos específicos no líquor ${ }^{28}$. Este achado, porém, deve ser interpretado com cautela, pois a quebra da barreira hemato-liquórica, por qualquer etiologia, poderá ser acompanhada de transferência passiva de anticorpos séricos para o líquor. Um estudo utilizando imunofluorescência indireta para pesquisa de anticorpos IgG, anti-T. gondii, no soro e líquor encontrou sensibilidade de $95 \%$ e $77 \%$ respectivamente ${ }^{28}$. O teste, entretanto, foi maisespeáfico no líquor do que no soro $(56 \% \times 30 \%)$, o que vem ao encontro dosresultadosaqui apresentados. A ausência de anticorpos IgG anti-T. gondii no líquor afastou o diagnóstico da neurotoxoplasmose em 92,3\% dosnossos casos, sendo um importante marcador para o diagnóstico diferencial da toxoplasmose cerebral na SIDA. AnticorposIgA, antiT. gondii, foram encontrados no líquor em $50 \%$ dos casos de neurotoxoplasmose, alcançando especificidade de $72,7 \%(p<0,05)$, podendo representar um marcador de auxílio para tal diagnóstico.

A saliva tem se mostrado um meio alternativo para pesquisa de anticorpos específicos, com implicações diagnósticas em determinadas infecções huma-

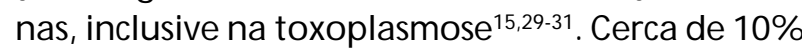
das proteínassalivares são imonoglobulinase, destas, $87 \%$ são da classe IgA que, como as IgM, são produzidas localmente. Já as IgG aí encontradas são derivadas, na sua maior totalidade, do plasma ${ }^{15,32,33}$. Durante a toxoplasmose aguda pode se detectar a presença do $T$. gondii na saliva, masnão em pacientes com SIDA e neurotoxoplasmose ${ }^{34}$. Estudos avaliando o valor diagnóstico de tal achado, bem como a demonstração de anticorpos específicos, são escassos. Nossos resultados demonstram boa correlação entre a presença de lgG, anti - T. gondii, na saliva e o diagnóstico de neurotoxoplasmose, com sensibilidade de $95,8 \%$, porém nenhuma correlação quando se avalia anticorpos IgA e IgM neste material.

Em conclusão, os resultados aqui apresentados enfatizam a importância dostestes sorológicospara o diagnóstico da neurotoxoplasmose em pacientescom SIDA, principalmente naquelescasosem que osachados de imagens são atípicosou com lenta resposta à terapêutica empiricamente instituída. A ausência de anticorpos IgG no soro e líquor depõem fortemente contra tal diagnóstico. Além disso, o encontro de IgA específica no líquor e IgG na saliva podem representar dois marcadores auxiliares para o diagnóstico da reativação da toxoplasmose a nível de SNC na SIDA.

\section{REFERÊNCIAS}

1. Bertoli F, Espino M, Arosemena JR, Fishback JL, Frenkel JK. A spectrum in the pathology of toxoplasmosis in patients with acquired immunodeficiency syndrome. Arch Pathol Lab Med 1995;119:214-224.

2. Dunlop O, Rootwelt V, Sannes M, et al. Risk of toxoplasmic encephalitis in AIDS patients: indications for prophylaxis. Scand J Infect Dis 1996; 28:71-73.

3. Frenkel JK, Hassenem KM, Hassenem RS, Brown E, Thulliez P, QuinteroNunez R. Transmission of Toxoplasma gondii in Panama city, Panama: a five year prospective cohort study of children, cats, rodents, birds and soil. Am J Trop Med Hyg 1995;53:458-468.

4. Garly M, Eskild P, Petersen C, Lundgren JD, Gerstoft J. Toxoplasmosis in Danish AIDS patients. Scand J Infect Dis 1997;29:597-600.

5. Zangerle R, Allergerger F, Pohl P, Fritsch P, Dierich MP. High risk of developing toxoplasmic encephalitis in AIDS patients seropositive to Toxoplasma gondii. Med Microbiol Immunol 1991;180:59-66.

6. Borges AS, Ferreira MS, Nishioka SA, Silvestre MTA, Silva AM, Rocha A. Agreement between premortem and postmortem diagnoses in patients with acquired immunodeficiency syndrome observed at a Brazilian teaching hospital. Rev Inst Med Trop São Paulo 1997;39:217-221.

7. Ferreira MS, Nishioka SA, Silvestre MT, Borges AS, Nunes-Araujo FR, Rocha A. Reactivation of Chagas' disease in patients with AIDS: report of three new cases and review of the literature. Clin Infec Dis 1997; 25:1397-1400.

8. Klatt EC, Nichols L, Nogughi TT. Envolving trends revealed by autopsies of patients with the acquired immunodeficiency syndrome. Arch Pathol Lab Med 1994;118:884-890.

9. Tuazon CV. Toxoplasmosis in AIDS patients. J Antimicrob Chemother 1989;23(Suppl):S77-S82.

10. Wanke C, Tuazon CV, Kovacs A, et al. Toxoplasma encephalitis in patients with acquired immune deficiency syndrome: diagnosis and response to therapy. Am J Trop Med Hyg 1987;36:509-516.

11. Luft BJ, Brooks RG, Conley FK, McCabe RE, Reminton JS. Toxoplasmic encephalitis in patients with acquired immunodeficiency syndrome. JAMA 1984;252:913-917.

12. Raffi F, Aboulker J, Michelet C, et al. A prospective study of criteria for the dagnosis of toxoplasmic encephalitis in 186 AIDS patients. AIDS 1997;11:177-184.

13. Darcy F, Foudrinier F, Mougeot G, et al. Diagnostic value of specific IgA antibodies in AIDS patients with Toxoplasma infection: a bicentric evaluation. Immunol Lett 1991;30:345-348.

14. Gross U, Roos T, Appoldt D, Heesemann J. Improved serological diagnosis of Toxoplasma gondii infection by detection of immunoglobulin $\mathrm{A}$ (IgA) and IgM antibodies against P30 by using immunoblot technique. J Clin Microbiol 1992;30:1436-1441

15. Hajeer AH, Balfour AH, Mostratos A, Crosse B. Toxoplasma gondii: 
detection of antibodies in human saliva and serum. Parasite Immunol 1994;16:43-50.

16. Patel B, Young Y, Duffy K, Tanner RP, Johnson J, Holliman RE. Immunoglobulin-A detection and the investigation of clinical toxoplasmosis. J Med Microbiol 1993;38:286-292.

17. Grant IH, Gold JWM, Rosenblum M, Niedzwiecki D, Armstrong D. Toxoplasma gondii serology in HIV-infected patients: the development of central nervous system toxoplasmosis in AIDS. AIDS 1990;4:519-521.

18. Hellerbrand C, Goebel FD, Disko R. High predictive value of Toxoplasma gondii IgG antibody levels in HIV-infected patients for diagnosis of cerebral toxoplasmosis. Eur J Clin Microbiol Infect Dis 1996;15:869-872.

19. Cimino C, Lipton RB, Williams A, Feraru E, Harris C, Hirschfeld A. The evaluation of patients with human immunodeficiency virus-related disorders and brain mass lesions. Arch Intern Med 1991;151:1381-1384.

20. Luft BJ, Hafner R, Korzun AH, et al. Toxoplasmic encephalitis in patients with the acquired immunodeficiency syndrome. N Engl J Med 1993; 329:995-1000

21. Haverkos HW. Assessment of therapy for toxoplasma encephalitis. Am J Med 1987;82:907-914.

22. Leport C, Raffi F, Matheron S, et al. Treatment of central nervous system toxoplasmosis with pyrimethamine/sulfadiazine combination in 35 patients with the acquired immunodeficiency syndrome. Am J Med 1988; 84:94-100.

23. Centers for Disease Control. Revision of the CDC surveillance case definition for acquired immunodeficiency syndrome. MMWR. 1987;36 (Suppl):S1.

24. Voller A, Bartlett A, Bidwell DE. Enzyme immunoassays with especial reference to ELISA techniques. J Clin Pathol 1978;31:507-520.

25. Ammassari A, Scoppettuolo G, Murri R. Changing disease patterns in focal brain lesion-causing disorders in AIDS. J Acquir Immune Defic Syndr Hum Retrovirol 1998;18:365-371.

26. Danneman BR, McCutchan JA, Israelski DM, et al. Treatment of toxoplasmic encephalitis in patients with AIDS: a randomized trial comparing pyrimethamine plus clindamycin to pyrimethamine plus sulfonamides. Ann Intern Med 1992;116:33-43.

27. Holliman RE. Clinical ande diagnostic findings in 20 patients with toxoplasmosis and the acquired immune deficiency syndrome. J Clin Microbiol 1991;35:1-4.

28. Wainstein MV, Wolffenbuttel L, Lopes D K, et al. Sensibilidade e especificidade do diagnóstico clínico, sorológico e tomográfico da encefalite por Toxoplasma gondii na síndrome da imunodeficiência adquirida. Rev Soc Bras Med Trop 1993;26:71-75.

29. Garcia MMA, Amorim MN, Viana LG, Garcia TCM, Katz N, Rabelo ALT. Detection of anti-Schistossoma antibodies in oral fluids. Mem Inst Oswaldo Cruz 1995;90:513.

30. Loyola AM, Durighetto AF Jr, Silva DAO, Mineo JR. Anti-Toxoplasma gondii immunoglobulins A and $\mathrm{G}$ in human saliva and serum. J Oral Pathol Med 1997;26:187-191.

31. Silva MV, Camargo ED, Vaz, AJ, Batista L. Immunodiagnosis of human leptospirosis using saliva. Trans R Soc Trop Med Hyg 1992;86:560-561.

32. Burns CA, Ebersole JL, Allansmith MR. Immunoglobulin A antibody leve1s in human tears, saliva and serum. Infect Immun 1982;36:1019-1022.

33. Grönblad EA. Concentration of immunoglobulins in human whole saliva: effect of physiological stimulation. Acta Odontol Scand 1981;40:87-95.

24. Amato-Neto V, Braz LMA, Jamra LMF, Higaki Y, Pasternak J. Pesquisa do Toxoplasma gondii na saliva de pacientes com síndrome da imunodeficiência adquirida (AIDS). Rev Hosp Clín Fac Med São Paulo 1990;45:171-172. 